Acta Cryst. (2002). A58 (Supplement), C215

\section{HARMONISING THERMODYNAMIC AND CRYSTALLOGRAPHIC INFORMATION WHEN MODELLING PHASES USING THE CALPHAD APPROACH}

S. Fries $^{1}$ B. Sundman ${ }^{2}$

${ }^{1}$ ACCESS e.V., RWTH-Aachen, D-52072 Aachen, Germany ${ }^{2}$ Dep. Mat. Sci. and Eng. Royal Institute of Technology, S-10044 Stockholm, Sweden

The development of multicomponent thermodynamic databases brings to evidence the necessity of crystallographic information in order to identify and model the many phases present. The combination of crystallographic and thermodynamic databases does not only help to solve the problem of naming phases but can also help to define some areas where a more systematic research on phase solubility ranges/stability/mechanisms of order/disorder, for instance, is needed.

To identify phases that although the different crystal structure are anyway related by some special lattice defect evolution (e.g. NiAs to $\mathrm{CdI}_{2}$-type, $\mathrm{Cu}_{9} \mathrm{Al}_{4}$-type to $\mathrm{Cu}_{5} \mathrm{Zn}_{8}$-type) or related by some distortions (NiAs to MnP-type) can simplify the thermodynamic model reducing the number of model parameters. Identification of families with an underlying lattice (e.g., fcc, bcc, hcp and their superlattices), allows to model order/disorder phenomena (e.g., fcc ordering in $\mathrm{Cu}-\mathrm{Au}$ and $\mathrm{Ni}-\mathrm{Al}$ ) using only a single Gibbs energy for both states, what brings more physical consistency to the modeling resulting even in descriptions of second order transitions (e.g. bcc ordering in $\mathrm{Cu}-\mathrm{Zn}$ ).

Only few of these connections are already identified and implemented in the thermodynamic databases. An extended and challenging task is in front of us and it requires the integration of the thermodynamic and crystallographic databases as well as expertise in both fields.

Our strategy supported by the Scientific Group Thermodata Europe (SGTE) is presented.

\section{Keywords: THERMODYNAMIC DATABASE , CALPHAD METHOD,} CRYSTALLOGRAPHIC DATABASE

\section{Acta Cryst. (2002). A58 (Supplement), C215}

\section{INDUSTRIAL PROBLEM SOLVING USING MULTIPLE DATABASES}

\section{J. Kaduk}

BP Chemicals P.O. Box 3011, Mc F-9 150 W. Warrenville Rd.

NAPER VILLE IL 60566 USA

Although the crystallographic databases are archives of atom coordinates, they contain additional information, which is useful in solving practical problems. A model for the structure of the corrosion deposit $\left(\mathrm{NH}_{4}\right) \mathrm{Fe}\left(\mathrm{CO}_{3}\right)(\mathrm{OH})_{2}(\mathrm{Cmcm}, a$ $=6.6154(6), b=12.0639(10)$, and $c=6.0263(5) \AA, Z=4)$ was found by searching the ICSD for compounds with C-centered orthorhombic cells and containing $\mathrm{C}, \mathrm{H}, \mathrm{N}$, and $\mathrm{O}$. Lower hydrates of magnesium chloride are sometimes isolated from preparations of polypropylene catalysts. Suitable models for $\mathrm{MgCl}_{2}\left(\mathrm{H}_{2} \mathrm{O}\right)_{4}(P 21 / c, a=5.9001(9), b=7.2709(7), c=8.4199(7)$ $\left.\AA, \beta=111.007(15)^{\circ}, \mathrm{Z}=2\right), \mathrm{MgCl}_{2}\left(\mathrm{H}_{2} \mathrm{O}\right)_{2}(C 2 / m, a=7.3886(29), b=$ 8.5498(24), $\left.c=3.6488(17) \AA, \beta=98.96(3)^{\circ}, \mathrm{Z}=2\right)$, and $\mathrm{MgCl}_{2}\left(\mathrm{H}_{2} \mathrm{O}\right)($ Pnma, $a$ $=8.899(7), b=3.6339(20), c=11.398(8) \AA, Z=4)$ were located by combining searches in the ICSD on chemistry and formula type. Monopotassium dihydrogen citrate, $\mathrm{KH}_{2} \mathrm{C}_{6} \mathrm{H}_{5} \mathrm{O}_{7}$, was identified using the PDF. The powder pattern could be indexed on a high-quality cell, and a $4 x$ super-cell was identified using the CDIF. This cell $(P-1, a=9.4537(2), b=13.0068(2), c=$ $14.9400(3) \AA, \alpha=109.882(1)^{\circ}, \beta=91.555(2)^{\circ}, \gamma=93.394(2)^{\circ}, \mathrm{V}=1722.34(6)$ $\AA^{3}, Z=8$ ) accounted for all of the peaks, and proved critical in determining the structure from twinned single crystals. Quantum chemical calculations were performed on all these structures to obtain quantitative understanding of the hydrogen bonding important in the structures.

\section{Keywords: DATABASES PROBLEM SOLVING INDUSTRIAL}

Acta Cryst. (2002). A58 (Supplement), C215

PROPERTIES COMPUTED AB INITIO FROM STRUCTURE

DATABASE ENTRIES: RESULTS AND PERSPECTIVES

Y. Le Page ${ }^{1}$ P.W. Saxe ${ }^{2}$ J.R. Rodgers ${ }^{3}$

${ }_{1}^{1}$ ICPET, National Research Council of Canada, Ottawa, Canada K1 A 0R

${ }^{2}$ Materials Design, Inc. , PO Box 1318, Angel Fire, NM 87710-131 ${ }^{3}$ Toth

Information Systems Inc., 2045 Quincy Ave., Ottawa, Canada K1J 6B

Quantum methods are becoming practical, affordable tools for mining structure databases for physical properties never measured before, increasing considerably the value of archived structure data. We have developed, and automated in point-and-click fashion, novel symmetry-general methods for extracting the $0 \mathrm{~K}$ elastic tensor coefficients from energy or stress calculated $a b$ initio using adequately strained atomic models derived from straight crystal structure entries in the CRYSTMET ${ }^{\circledR}$ and ICSD ${ }^{\circledR}$ databases. Results including the pressure-dependence of their elastic coefficients down to lower-mantle pressures will be presented for a wide variety of materials spanning all crystal systems and ranging in computational complexity from diamond $(F d-3 m, 2$ atoms per primitive cell, 5 minutes) to tourmaline ( $r 3 m, 53$ atoms per primitive cell, one week computation on a single PC). The quality of numbers obtained with the VASP quantum engine helped identify and resolve several issues in published experimental stiffness tensor data. Simultaneous serial execution of many tasks, one per task server (networked ghz pcs with large geographical extension monitored from a central job server), leads to affordable setups with flexibility, reliability and throughput that compare with those of Beowulf clusters or supercomputers.

About a dozen thermomechanical properties of the equivalent isotropic material like Young's modulus, the shear modulus, the bulk modulus, the speed of sound for longitudinal and transverse waves etc... are directly calculable from the stiffness tensor. The blueprint of our automated computation of the stiffness tensor can be adapted to calculation of other intrinsic bulk properties at $0 \mathrm{~K}$. Extension to finite temperatures will be discussed.

Keywords: ELASTICITY AB INITIO STRUCTURE DATABASES

\author{
Acta Cryst. (2002). A58 (Supplement), C215 \\ COMPUTED DATA IN THE DESIGN OF MATERIALS \\ E. Wimmer \\ Materials Design Sarl 44, Avenue F.-A. Bartholdi LE MANS 72000 FRANCE
}

The dramatic progress in computational science has made it possible to predict a wealth of properties including crystallographic structures, thermodynamic and thermomechanical data, as well as electronic, optical and magnetic properties at a remarkable level of accuracy and efficiency. In some cases such as surfaces, interfaces, and point defects in solids, computational approaches are possibly the most reliable source of property data. The present scientific and technological trends indicate that the pace of this development is likely to be sustainable for the coming decades. Given the fact that computations are inherently easier to automate than experimental procedures, we are facing a tremendous augmentation in our ability to generate data of high quality and integrity. This situation raises a number of scientific and technological issues including (i) the reliability of computed data, (ii) the decision on which data should be stored, (iii) the best format for storing the data, and (iv) the best way to make these data available. This contribution shows that one of the most critical aspects is a close integration of experimental and computed data. On the one hand, one needs a sufficient overlap between experimental and computed data to allow a thorough validation of computed (and perhaps in the future also measured) data. On the other hand, one should exploit the complementarity between experimental and computed data to allow a more comprehensive understanding of materials. The above points will be illustrated by the prediction of surface properties as related to heterogeneous catalysis, optoelectronic properties and thermomechanical properties.

Keywords: COMPUTATIONS MATERIALS DESIGN 